\title{
Ciliates display promise for foreign gene expression
}

\section{Clifford F. Brunk}

The study of Tetrahymena thermophila, the simple ciliate of introductory biology laboratory exercises, has led to a remarkable series of discoveries in biology, many of which have found important applications in biotechnology. These range from the identification of ribozymes and telomerase to the first example of a noncanonical nuclear genetic code and the role of histone acetyltransferase A in gene activation ${ }^{1-4}$. Now this remarkable ciliate has made the leap from mere model organism in basic biology to a new role as a potentially useful expression system in biotechnology. As Tetrahymena is nonpathogenic, has a very short generation time, and can be grown to high cell density in inexpensive media, it is an ideal candidate system for production of pharmaceuticals and vaccines.

When transformation of an expression system with a foreign gene does not produce a selectable phenotype, identification of transformed cells and the maintenance of the transformed state can be tricky, if not impossible. In this issue, Gaertig et al. describe the engineering of a Tetrahymena expression system that gets around this problem by exploiting the drug sensitivity of a particular mutant strain $^{5}$. A mutation in either one of the two Tetrahymena $\beta$-tubulin genes-a substitution of Lys350 by methionine-produces sensitivity to the microtubule drug, paclitax$\mathrm{el}^{7}$, and knocking out the mutant $\beta$-tubulin gene restores resistance (see Fig. 1). Thus, if a foreign gene is targeted to the mutant $\beta$ tubulin gene by homologous recombination, transformed cells can be selected by their resistance to paclitaxel and the foreign gene is expressed under the control of the $\beta$-tubulin promoter. In one fell swoop, an introduced foreign gene is expressed and produces a selectable phenotype. This approach is not unique to Tetrahymena and should be applicable to other systems.

This system has been used to express a surface immobilization antigen (i-antigen) from the parasitic cousin of Tetrahymena, the ciliate Ichthyophthirius multifiliis, which causes "Ich" or "white spot" disease in economically important freshwater fish ${ }^{6}$. The iantigen of I. multifiliis is apparently responsible for protective immunity and is thus a prime candidate for vaccine development ${ }^{8}$. Unfortunately, however, I. multifiliis can only be grown in association with its host, making

Clifford F. Brunk is professor in the Biology Department, University of California, Los Angeles, 90024-1606 (cbrunk@ucla.edu).

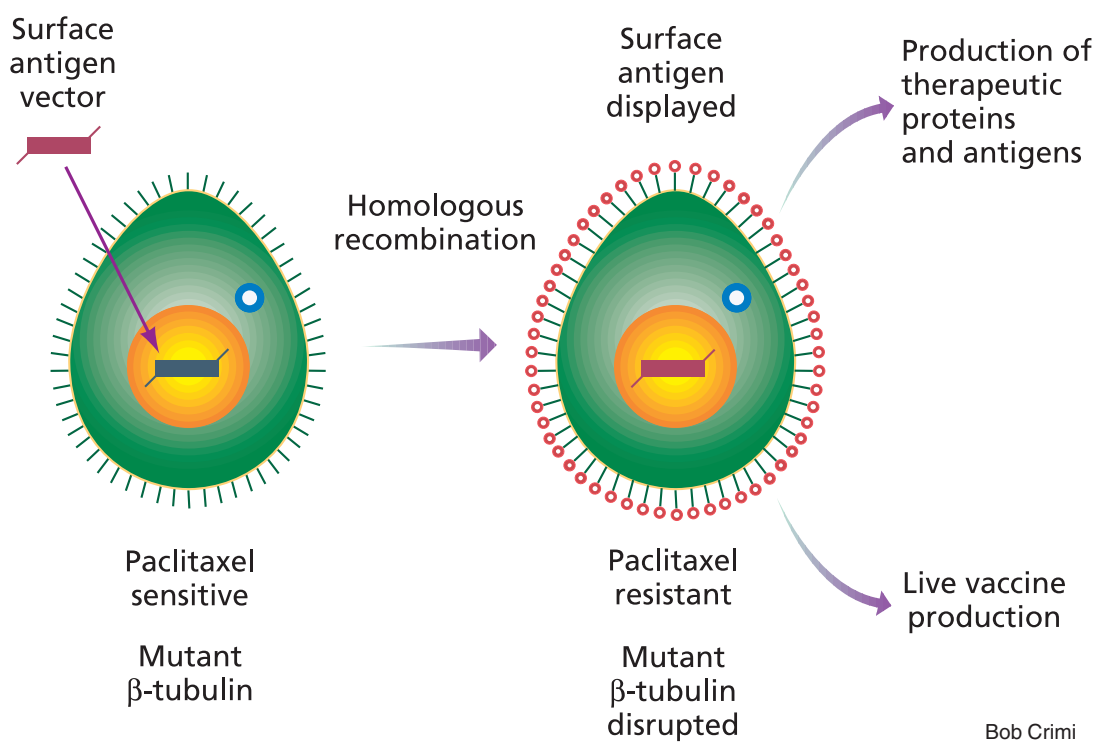

Figure 1. Tetrahymena expressing a mutant copy of the $\beta$-tubulin gene (btu1-1K350M) are paclitaxel sensitive. When transformed with an Ichthyophthirius multifiliis i-antigen flanked by the $5^{\prime}$ and $3^{\prime}$ regions of the $\beta$-tubulin gene, the mutant gene is disrupted, the cells are paclitaxel resistant, and the i-antigen is expressed and displayed on the cell surface.

it difficult to obtain sufficient quantities of iantigen protein ${ }^{9}$. Moreover, like most ciliates, I. multifiliis employs a noncanonical nuclear genetic code, in which codons UAA and UAG specify glutamine rather than chain termination $^{10}$. This means that any attempt to translate I. multifiliis genes in a conventional expression system employing a canonical nuclear genetic code will generally produce premature termination and result in nonfunctional truncated proteins. Tetrahymena conveniently shares the same noncanonical nuclear genetic code used by I. multifiliis, allowing accurate translation of the parasite's proteins in Tetrahymena. Of course, the noncanonical system used by Tetrahymena may create problems when it is used to translate genes from organisms using the canonical nuclear genetic code that terminates with a UAA or UAG codon. That said, among mammalian genes found in GenBank, UGA appears more frequently than UAA and UAG termination codons combined ${ }^{11}$. When a foreign gene terminating with either UAA or UAG is translated in Tetrahymena, the resulting protein is extended at the $\mathrm{C}$ terminus by a polypeptide corresponding to the sequence between the normal termination codon and the next in-frame UGA. These additional Cterminal amino acids are unlikely to interfere with the function of the protein, however. Thus, not only is the Tetrahymena system capable of translating foreign genes using the noncanonical nuclear genetic code, it should also produce active proteins from many foreign genes using the canonical nuclear genetic code.

Importantly, the $\mathrm{i}$-antigen is expressed in Tetrahymena at the cell surface. Probing Tetrahymena with a fluorescently labeled antiserum directed against the i-antigen reveals strong labeling of both oral and somatic cilia in a pattern very similar to that observed in I. multifiliis. Moreover, antiserum directed against $I$. multifiliis i-antigen causes immediate immobilization of transformed Tetrahymena (by cross-linking cilia). The surface localization of the i-antigen suggests that its signal peptide is functioning appropriately in Tetrahymena. It remains to be seen, however, whether proteins from more distantly related organisms will also be appropriately localized when expressed in Tetrahymena.

The expression of I. multifiliis i-antigen in Tetrahymena makes possible the large-scale production of this protein as a potential vaccine against $I$. multifiliis. The high cell density to which Tetrahymena can be grown suggests that the i-antigen may be produced on the order of milligrams per liter. Furthermore, its display on the cell membrane opens the possibility that transformed Tetrahymena organisms could be used as a live vaccine against $I$. multifiliis. Since Tetrahymena are normally present in fresh- 
water ecosystems, the introduction of quantities of transformed cells displaying the $I$. multifiliis i-antigen in their cell membranes may immunize fish against the parasitic $I$. Multifiliis. Whether or not the use of Tetrahymena as a living vaccine against "white spot" disease becomes a reality, the innovative system described by Gaertig et al. for the identification and maintenance of transformed cells expressing foreign proteins that do not confer a selectable phenotype has real merit and is not for ciliates only. 1. Cech, T.R. Ann. Rev. Biochem. 59, 543-568 (1990).
2. Blackburn, E.H. \& Szostak, J.W. Ann. Rev. Biochem.
53, 163-194 (1984).
3. Horwitz, S. \& Gorvosky, M.A. Proc. Natl. Acad. Sci.
USA 82, 2452-2455 (1985).
4. Brownell, J. E. et al. Cell 84, 843-851 (1996).
5. Gaertig, J. et al. Nature Biotechnol. 17, 462-465 (1999).
6. Matthews, R.A. in Parasitic diseases of fish (eds Pike, A.W. \& Lewis, J.W.) 17-42 (Samara, Dyfed, UK; 1994).

7. Gaertig, J. et al. Proc. Natl. Acad. Sci. USA 91, 4549-4553 (1994).

8. Clark, T.G. et al. Annu. Rev. Fish Dis. 5, 113-131 (1996).

9. Noe, J.G. \& Dickerson, H.W. J. Parasitol. 81, 1022-1024 (1995).

10. Clark, T.G. et al. Proc. Natl. Acad. Sci. USA 89 , 6363-6367 (1992).

11. Codon Usage Database: http://www.dna. affrc.go.jp/ nakamura/codon.html

\section{Spying on the hidden lives of proteins}

\section{Richard N. Day and David W. Piston}

Fluorescence microscopy studies of protein localization in the cell have contributed enormously to our understanding of biological processes. Since the introduction of immunofluorescence techniques more than 50 years ago, however, most fluorescence microscopy procedures have changed very little. Rapid advances in technology have led to the development of many new imaging modalities, but most of these have found little general use in biomedical research and languish in the labs of the developers. In the March 26 issue of Science, $\mathrm{Ng}$ and colleagues ${ }^{1}$ show us how a combination of two advanced imaging methods, fluorescence resonance energy transfer (FRET) and fluorescence lifetime imaging microscopy (FLIM), may revolutionize the way immunofluorescence is performed. In their study, the authors use these fluorescence microscopy techniques to acquire a spatial map of the distribution of the activated form of the enzyme, protein kinase $\mathrm{C} \alpha(\mathrm{PKC} \alpha)$ in intact cells.

The spatial map of a cell acquired by conventional fluorescence microscopy is limited to about $0.2 \mu \mathrm{m}(200 \mathrm{~nm})$, the resolution of the optical imaging system. A substantial improvement in the spatial resolution of the microscope can be achieved by detecting FRET, a quantum mechanical process by which excited-state energy is transferred from a donor fluorophore to an acceptor molecule in close proximity (Fig. 1). Because the efficiency of FRET decreases rapidly as the distance between the two fluorophores increases,

Richard N. Day is associate professor Departments of Medicine and Cell Biology, National Science Foundation Center for Biological Timing, University of Virginia, Charlottesville, VA 22908

(rnd2v@virginia.edu).David W. Piston is associate professor Department of Molecular Physiology and Biophysics, Vanderbilt University, Nashville, TN 37232 (dave.piston@mcmail.vanderbilt.edu).
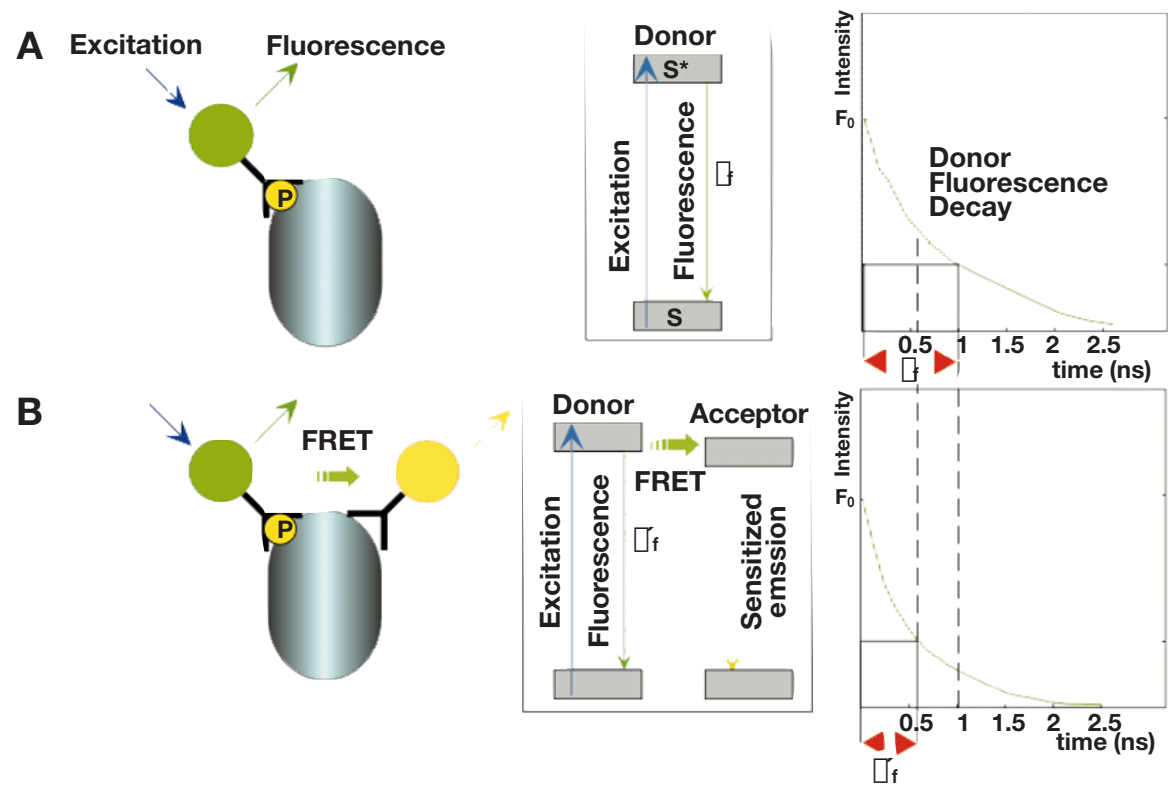

Figure 1. Detection of fluorescence resonance energy transfer (FRET) by fluorescence lifetime imaging microscopy (FLIM). (A) Activated form of a protein labeled with an antibody conjugated to a donor fluorophore. On absorbing light, the fluorophore changes from ground state (S) to the excited state $\left(S^{\star}\right)$, as illustrated in the simplified Jablonski energy-level diagram (A, middle). This is followed by emission of a photon (fluorescence) during the next few nanoseconds. The exponential decay of fluorescence emission intensity determines the donor fluorescence lifetime ( $A$, right, $\tau f)$. (B) The protein labeled with both donor and acceptor fluorophores, illustrating the effect of energy transfer on donor fluorescence lifetime. As the Jablonski diagram ( $B$, middle) shows, deactivation from the donor excited state can occur by fluorescence or through the radiationless transfer of energy to the acceptor by FRET. The occurrence of FRET is detectable by a decrease in the donor fluorescence lifetime (B, right, $\tau$ ' $f$ ).

energy transfer occurs only when the two are within about $5 \mathrm{~nm}$ of each other, roughly the size scale of a $35 \mathrm{kDa}$ spherical protein. The detection of FRET would thus seem to be an ideal way to spy on intracellular events such as protein-protein interactions.

In practice, however, the use of FRET microscopy is limited by problems of labeling specificity, probe concentration, and photobleaching ${ }^{2}$. These latter two limitations can be overcome by detecting FRET through its effect on the fluorescence lifetime of the donor fluorophore using FLIM. The average excited-state lifetime of a fluorescent mole- cule is fleetingly short, typically less than 10 ns, and is critically dependent on the local environment surrounding the probe ${ }^{3,4}$. The FLIM technique detects a spatial lifetime map of these nanosecond decay kinetics, and therefore can reveal localized variations in the probe environment, in addition to a simple picture of fluorophore localization ${ }^{4,5}$. Since FRET occurs during the excited-state lifetime of the donor molecule, it significantly affects the donor fluorescence lifetime. The change in lifetime due to the presence of an acceptor fluorophore in the donor's local environment can be detected by FLIM (Fig. 